\title{
Is total colectomy for colorectal cancer contraindicated in elderly patients?
}

\author{
S Grassia, C La Tessa*, S Spiezia, R Romagnuolo, N Carlomagno, C Dodaro, A Renda \\ From XXIII Annual Meeting of the Italian Society of Geriatric Surgery \\ Lecce, Italy. 2-4 December 2010
}

\section{Background}

More and more frequently elderly patients are operated on for colorectal cancer (CRC). Total colectomy is indicated in selected cases such as synchronous tumors, cancer in FAP, HNPCC, emergency. We evaluated the impact of the age risk factor on patients' outcome after total colectomy for cancer.

\section{Methods}

We reviewed our series concerning 27 patients submitted to total colectomy for CRC between January 2000 and September 2010. Indications were: synchronous tumors, cancer in FAP, HNPCC, and emergency. We divided them into two groups according to their age: $11(40.7 \%)<65$ years (Group A) and $16(59.3 \%) \geq$ 65 years (Group B). For both groups the following preoperative parameters were investigated: emergency/elective surgery, comorbidity, smoke abuse, ASA score, and surgical operation. Postoperative course related to systemic (pulmonary, cardiac, renal and liver failure, pulmonary embolism, urinary tract infections) and specific surgical complications (anastomotic dehiscence, hemorrhage, postoperative anemia, wound infection, prolonged ileus $>3$ days) were compared in two groups.

\section{Results}

Comorbidity was higher in older patients. We found a different distribution of ASA scores in the two groups: in the first group $5(45.5 \%)$ patients had score $\leq 2$, while $6(54.5 \%)$ patients presented score $\geq 3$; in the second group $10(62.5 \%)$ patients presented score 3 , while 6 (37.5\%) patients had score 4.

\footnotetext{
* Correspondence: cristinalatessa@gmail.com

Surgical, Anaesthesiology-Rianimative and Emergency Science Department, University of Naples "Federico II", Italy
}

We performed $2(7.4 \%)$ total proctocolectomy and permanent ileostomy; 1 (3.7\%) restorative proctocolectomy with ileal pouch-anal anastomosis (IPAA); 24 (88.9\%) total colectomy and ileo-rectal anastomosis (IRA). We observed 1 hemorrhage in a patient belonging to group A with ASA score 2. Systemic complications were higher in group B. We did not observe significant differences for surgical complications but for postoperative anemia (more frequent in group B). Average hospital stay was similar (11.2 days vs 12.8 days). Mortality [2/27 (7.4\%), in group B (2/16-12.5\%)] was not statistically significant.

\section{Conclusions}

After total colectomy for CRC we observed slightly higher postoperative morbidity and mortality in elderly patients. However our morbidity, mostly due to systemic complications, was acceptable and depended on a greater presence of preoperative risk factors. In conclusion we believe that, after an accurate preoperative evaluation, the age is not a contraindication for total colectomy.

\section{Published: 24 August 2011}

\section{References}

1. Marusch F, Koch A: The impact of the risk factor "age" on the early postoperative results of surgery for colorectal carcinoma and its significance for perioperative management. World J Surg 2005, 29(8):1013-21, discussion 1021-2.

2. Longo WE, Virgo KS: Risk factors for morbidity and mortality after colectomy for colon cancer. Dis Colon Rectum 2000, 43(1):83-91.

doi:10.1186/1471-2318-11-S1-A24

Cite this article as: Grassia et al:: Is total colectomy for colorectal cancer contraindicated in elderly patients? BMC Geriatrics 2011 11(Suppl 1):A24.

\section{Biomed Central}

(C) 2011 Grassia et al; licensee BioMed Central Ltd. This is an open access article distributed under the terms of the Creative Commons Attribution License (http://creativecommons.org/licenses/by/2.0), which permits unrestricted use, distribution, and reproduction in any medium, provided the original work is properly cited. 